\title{
Erratum to: Genetic Association Between KIBRA Polymorphism and Alzheimer's Disease with in a Japanese Population
}

Eri Kawai $^{1}$ - Nobuto Shibata ${ }^{1}$ Tomoyuki Nagata ${ }^{2,3} \cdot$ Shunichiro Shinagawa $^{2}$. Kenji Tagai $^{2} \cdot$ Tohru Ohnuma $^{1} \cdot$ Hiromi Shimazaki ${ }^{1} \cdot$ Aiko Toda $^{1}$.

Koji Kasanuki $^{1}$ - Toshiki Takayama ${ }^{1}$ Ayako Suzuki ${ }^{1} \cdot$ Kazuhiko Nakayama $^{2}$. Hisashi Yamada $^{3} \cdot$ Heii Arai $^{1}$

Published online: 21 April 2015

(C) Springer Science+Business Media New York 2015

\section{Erratum to: Neuromol Med}

DOI 10.1007/s12017-015-8348-8

The original publication contained an error in the fifth co-author's name. It should have read as Kenji Tagai. The authors regret the inconvenience caused to the readers in this regard.

The online version of the original article can be found under doi:10.1007/s12017-015-8348-8.

Nobuto Shibata

nshibata@juntendo.ac.jp

1 Department of Psychiatry, Juntendo University School of Medicine, 2-1-1 Hongo, Bunkyo-ku, Tokyo 113-8421, Japan

2 Department of Psychiatry, Jikei University School of Medicine, Tokyo, Japan

3 Division of Molecular Genetics, Institute of DNA Medicine, Jikei University School of Medicine, Tokyo, Japan 